\title{
Asiago eclipsing binaries program
}

\author{
III. V570 Persei $i^{\star, \star \star}$ \\ L. Tomasella ${ }^{1}$, U. Munari ${ }^{1}$, S. Cassisi ${ }^{2}$, A. Siviero ${ }^{1}$, S. Dallaporta ${ }^{3}$, R. Sordo ${ }^{1}$, and T. Zwitter ${ }^{4}$ \\ 1 INAF Osservatorio Astronomico di Padova, Sede di Asiago, 36012 Asiago (VI), Italy \\ e-mail: lina.tomasella@oapd.inaf.it \\ 2 INAF Osservatorio Astronomico di Collurania, via M. Maggini, 64100 Teramo, Italy \\ 3 Via Filzi 9, 38034 Cembra (TN), Italy \\ ${ }^{4}$ University of Ljubljana, Department of Physics, Jadranska 19, 1000 Ljubljana, Slovenia
}

Received 21 December 2007 / Accepted 4 March 2008

\section{ABSTRACT}

\begin{abstract}
The orbit and physical parameters of the previously unsolved double-lined eclipsing binary V570 Per, discovered by the Hipparcos satellite, were derived using high-resolution Echelle spectroscopy and $B, V$ photoelectric photometry. The metallicity from $\chi^{2}$ analysis of the spectra is $[\mathrm{M} / \mathrm{H}]=+0.02 \pm 0.03$, and reddening from interstellar NaI and KI absorption lines is $E_{B-V}=0.023 \pm 0.007$. V570 Per is a well-detached system, with shallow eclipses (due to low orbital inclination) and no sign of chromospheric activity. The two components have masses of $1.449 \pm 0.006$ and $1.350 \pm 0.006 M_{\odot}$ and spectral types F3 and F5, respectively. They are both still within the main sequence band $\left(T_{1}=6842 \pm 25 \mathrm{~K}, T_{2}=6562 \pm 25 \mathrm{~K}\right.$ from $\chi^{2}$ analysis, $R_{1}=1.523 \pm 0.030, R_{2}=1.388 \pm 0.019 R_{\odot}$ derived by forcing the orbital solution to conform to the spectroscopic light ratio) and are dynamically relaxed to co-rotation with the orbital motion $\left(V_{\mathrm{rot}, 1,2} \sin i=40\right.$ and $\left.36( \pm 1) \mathrm{km} \mathrm{s}^{-1}\right)$. The distance to V570 Per obtained from the orbital solution is $123 \pm 2 \mathrm{pc}$, in excellent agreement with the revised Hipparcos distance of $123 \pm 11 \mathrm{pc}$. The observed properties of V570 Per components were compared to available families of stellar evolutionary tracks and, in particular, to BaSTI models computed on purpose for exactly the observed masses and varied chemical compositions. This system is interesting since both components have their masses in the range where the efficiency of convective core overshooting has to decrease with the total mass as a consequence of the decreasing size of the convective core during the central H-burning stage. Our numerical simulations show that, in order to match all empirical constraints, a small but not null overshooting is required, with efficiencies of $\lambda_{\mathrm{OV}}=0.14$ and 0.11 for the 1.449 and $1.350 M_{\odot}$ components, respectively. This confirms the finding of Paper II on the similar system V505 Per. At the $\approx 0.8$ Gyr age of the system, the element diffusion has reduced the surface metallicity of the models from the initial $[\mathrm{M} / \mathrm{H}]=+0.17$ to $[\mathrm{M} / \mathrm{H}]=+0.02$, in perfect agreement with the spectroscopically derived $[\mathrm{M} / \mathrm{H}]=+0.02 \pm 0.03$ value.
\end{abstract}

Key words. stars: fundamental parameters - binaries: spectroscopic - binaries: eclipsing - stars: individual: V570 Per

\section{Introduction}

In the present series of papers we derive global physical parameters for a selection of double-lined eclipsing binaries (SB2 EBs) by means of Echelle high-resolution, high- $S / N$ spectroscopy and $B, V$ photoelectric photometry. Orbital solutions provide masses and radii, reddening is measured from intensity of interstellar absorption lines, and atmospheric analysis supplies effective temperatures, surface gravities, metallicity, and rotational velocities. The physical parameters we derive are of high quality (1\% accuracy regime) and are used to constrain the input physics of theoretical stellar models. We compute tailored evolutionary stellar models for the exact masses and chemical mixtures observed in the components of the binaries. This allows better focus on the effect expected, for example, from overshooting and element diffusion. Siviero et al. (2004) and Tomasella et al. (2008), hereafter Papers I and II, outline details of the type of data and methods used throughout this program.

$\star$ Based mainly on data obtained with Asiago $1.82 \mathrm{~m}$ telescope.

$\star \star$ Table 1 is only available in electronic form at the CDS via anonymous ftp to cdsarc.u-strasbg.fr (130.79.128.5) or via http://cdsweb.u-strasbg.fr/cgi-bin/qcat?J/A+A/483/263
V570 Per (HD 19457, HIP 14673) is a nearby eclipsing binary of early $\mathrm{F}$ spectral type, whose variability, characterized by a 1.9-day period, was discovered by the Hipparcos satellite. Its components do not show intrinsic variability, they are well-separated, perfectly round, slow rotating, and well within their Roche lobes, which makes them proper tests for stellar models. Though seen projected toward the $\alpha$ Per (Melotte 20) young open cluster, V570 Per is a foreground star not physically associated to the cluster. This can be deduced from the comparison between the distance and proper motion of the cluster $\left(d=183 \pm 7 \mathrm{pc}, \mu_{\alpha}^{*}=+22.47 \pm 0.16 \mathrm{mas} \mathrm{yr}^{-1}\right.$,

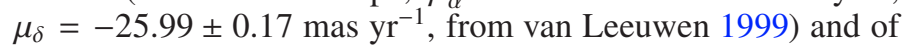
V570 Per $\left(d_{\text {HIP } 1997}=117 \pm 14 \mathrm{pc}, \mu_{\alpha}^{*}=+52.20 \pm 0.85 \mathrm{mas} \mathrm{yr}^{-1}\right.$,

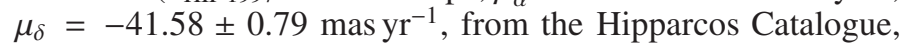
ESA 1997). The revised Hipparcos distance to V570 Per is $d_{\text {HIP } 2007}=123 \pm 11$ pc $($ van Leeuwen 2007).

A preliminary photometric and spectroscopic study of V570 Per was presented by Munari et al. (2001, hereafter M01). It was derived during an evaluation of the performances expected from Gaia (an ESA Cornerstone mission) on eclipsing binaries, and as such, the accuracy and abundance of input observational data (Hipparcos/Tycho photometry and Gaia-like ground-based 


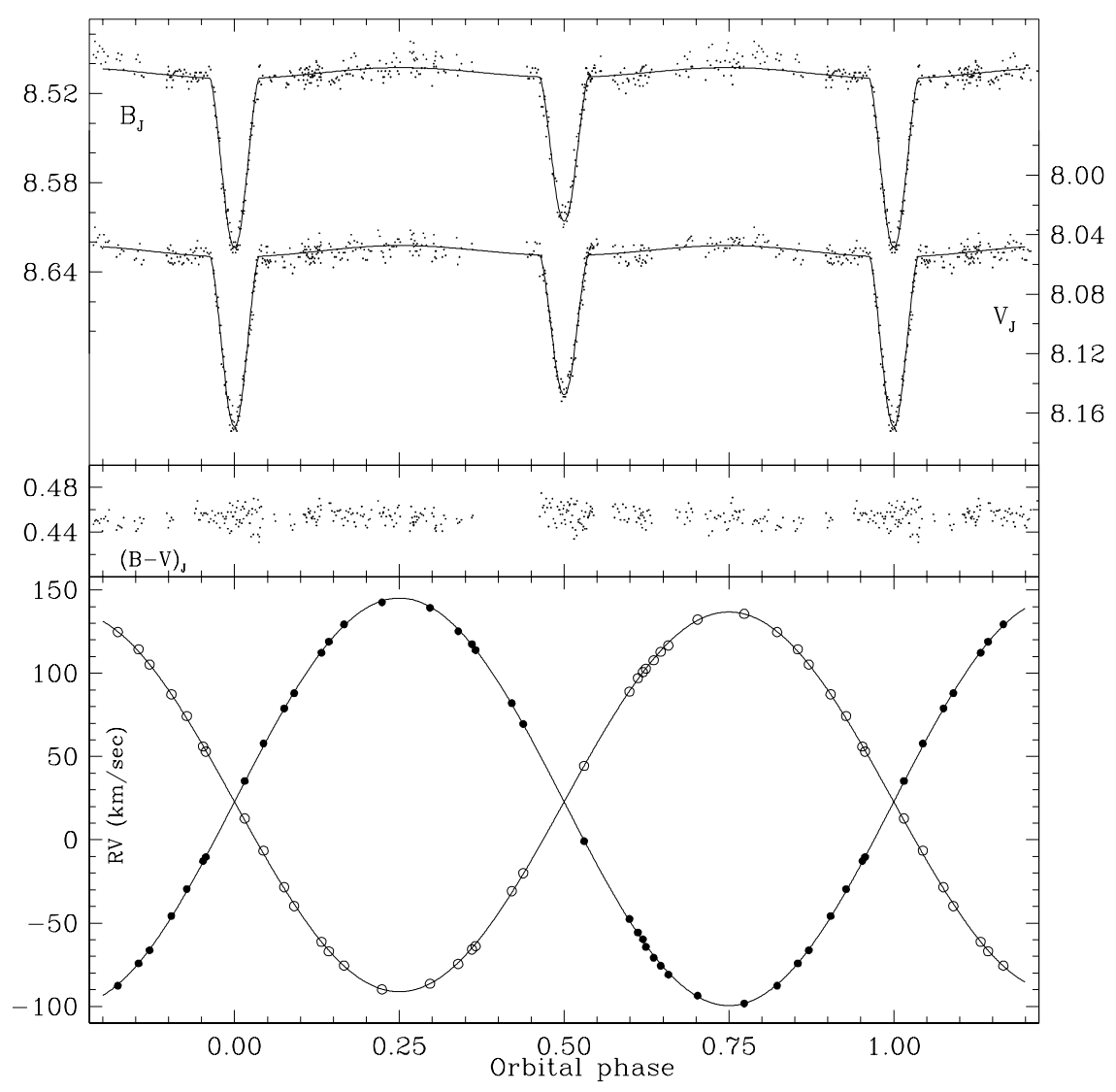

Fig. 1. The observed $B, V, B-V$, and radial velocity curves of V570 Per. In the radial velocity panel, the open circles indicate the hotter and more massive (primary) star, while the filled circles pertain to the cooler and less massive (secondary) star. The orbital solution from Table 3 is over-plotted to the observed data.

spectra) were necessarily lower than in the present study. A new study of the binary based on a much improved set of observational data is therefore justified, especially if supported by brand new reddening and atmospheric analysis.

\section{Photometric data}

The photometric observations of V570 Per were obtained in $B$ and $V$ (standard Johnson filters) with a $28 \mathrm{~cm}$ SchmidtCassegrain telescope and an Optec SSP5 photometer. The diaphragm has a diameter of 77 arcsec. There are no stars in the aperture brighter than $V=15.5 \mathrm{mag}$ (contributing less than 0.001 mag to recorded photometry). The instrumentation already proved to be very accurate and reliable (cf. Papers I and II) and thus perfectly suited to dealing with the low-amplitude eclipses of V570 Per (cf. Fig. 1).

The comparison star is HD $19805\left(\right.$ HIP $14980, B_{\mathrm{T}}=8.108 \pm$ $0.015, V_{\mathrm{T}}=7.973 \pm 0.012$, spectral type $\left.\mathrm{B} 9.5 \mathrm{~V}\right)$ and the check star is TYC $33153081\left(B_{\mathrm{T}}=9.919 \pm 0.029, V_{\mathrm{T}}=9.567 \pm 0.032\right)$. Both the comparison and check stars are close to V570 Per on the sky (the distances being $\sim 42$ and $\sim 18$ arcmin, respectively) so the atmospheric corrections are rather small. All the observations were obtained at heights over the horizon in excess of 30 degrees. The comparison star was measured against the check star at least once each observing run. In all, 34 measurements of the magnitude difference comparison-check star were collected. It remained constant throughout the whole observing campaign within a standard deviation of 0.006 mag. This confirms Hipparcos findings that both the comparison and the check stars are not variable, and thus well-suited to serving in the photometry of V570 Per. Following the Bessell (2000) transformations from the Tycho to Johnson photometric system, we adopted $B=8.073$ and $V=7.957$ for the comparison star.

In all, 446 measurements in $B$ and 465 in $V$ were collected for V570 Per between 2000 and 2003. They are listed in Table 1 (available electronic only). Each photometric point is actually the mean of 10 consecutive and independent measurements (every one lasting $5 \mathrm{~s}$ ), and the typical error of the mean is $0.006 \mathrm{mag}$ in $B$ and $0.005 \mathrm{mag}$ in $V$. All the observations are corrected for atmospheric extinction and color equation via nightly calibration on Landolt's equatorial fields.

The light curves of V570 Per in each band, as well as the $(B-V)$ color, are shown in Fig. 1. Spectroscopic and photometric observations are well-distributed in phase. The eclipses are very shallow due to the low inclination of the orbit $\left(i=77^{\circ}\right)$, which is the main reason for the absence of color variations during the eclipses (both stars remain essentially visible throughout the whole eclipses). The mean brightness out of eclipses is $B=8.505$ and $V=8.053 \mathrm{mag}$. The dispersion of the $B$ and $V$ measurements about their mean value out of the eclipses ( $\left.\sigma_{B}=0.007, \sigma_{V}=0.006\right)$ is only marginally higher than the accuracy of a single measurement. Thus any intrinsic variability of the amplitude larger than 0.007 mag should be ruled out.

\section{Spectroscopic observations}

The spectra of V570 Per were obtained in 1999-2002 with the Echelle+CCD spectrograph on the $1.82 \mathrm{~m}$ telescope operated by INAF Osservatorio Astronomico di Padova atop Mt. Ekar (Asiago). The instrumentation and observing set-up match those 
Table 2. Measured radial velocities $\left(\mathrm{km} \mathrm{s}^{-1}\right)$ of V570 Per.

\begin{tabular}{|c|c|c|c|c|c|c|c|c|c|}
\hline \multirow[t]{2}{*}{ \# } & \multirow[t]{2}{*}{ HJD } & \multirow[t]{2}{*}{ Phase } & \multicolumn{3}{|c|}{ Star 1} & \multicolumn{3}{|c|}{ Star 2} & \multirow[t]{2}{*}{$<\mathrm{S} / \mathrm{N}\rangle$} \\
\hline & & & $\mathrm{RV}_{\odot}$ & $\epsilon$ & $\mathrm{O}-\mathrm{C}$ & $\mathrm{RV}_{\odot}$ & $\bar{\epsilon}$ & $\mathrm{O}-\mathrm{C}$ & \\
\hline 30716 & 153.4401 & 0.773 & +135.7 & 1.0 & 0.10 & -98.2 & 0.6 & 0.04 & 121 \\
\hline 30796 & 154.5557 & 0.360 & -65.8 & 0.6 & -0.78 & +117.5 & 0.5 & 0.40 & 112 \\
\hline 30842 & 155.4359 & 0.823 & +124.8 & 1.0 & -0.20 & -87.6 & 0.5 & -0.73 & 126 \\
\hline 30903 & 156.4674 & 0.366 & -63.8 & 0.5 & -1.58 & +113.9 & 0.4 & -0.20 & 121 \\
\hline 31161 & 165.4203 & 0.075 & -28.4 & 0.5 & 0.54 & +78.9 & 0.6 & 0.56 & 130 \\
\hline 31219 & 166.4546 & 0.619 & +100.7 & 0.5 & 0.40 & -59.7 & 0.3 & 0.66 & 143 \\
\hline 31268 & 167.4285 & 0.132 & -61.2 & 0.8 & 0.06 & +112.4 & 0.5 & -0.60 & 128 \\
\hline 31317 & 169.5041 & 0.224 & -89.8 & 0.6 & -0.14 & +142.6 & 1.2 & -0.90 & 130 \\
\hline 31456 & 197.4553 & 0.928 & +74.3 & 0.3 & 1.66 & -29.6 & 0.8 & 1.06 & 137 \\
\hline 31779 & 206.3355 & 0.599 & +89.0 & 0.5 & -0.23 & -47.5 & 0.9 & 0.97 & 133 \\
\hline 31821 & 209.3144 & 0.166 & -75.5 & 0.9 & 0.17 & +129.3 & 1.1 & 0.80 & 105 \\
\hline 31967 & 217.4346 & 0.438 & -20.0 & 0.7 & 0.48 & +69.6 & 0.5 & 0.33 & 110 \\
\hline 32080 & 225.3691 & 0.612 & +97.0 & 0.8 & 0.43 & -55.7 & 0.4 & 0.64 & 117 \\
\hline 33297 & 480.5559 & 0.854 & +114.4 & 0.6 & 1.10 & -74.2 & 0.8 & 0.11 & 110 \\
\hline 33318 & 485.4339 & 0.420 & -30.8 & 0.2 & 1.31 & +82.0 & 0.5 & 0.26 & 102 \\
\hline 33378 & 505.4620 & 0.956 & +52.9 & 0.7 & -1.02 & -10.3 & 0.9 & 0.28 & 113 \\
\hline 33431 & 507.2637 & 0.904 & +87.3 & 0.5 & -0.17 & -45.8 & 0.5 & 0.78 & 123 \\
\hline 33439 & 507.3552 & 0.952 & +56.1 & 0.4 & -0.57 & -12.7 & 0.6 & 0.82 & 137 \\
\hline 33706 & 533.4932 & 0.702 & +132.2 & 0.8 & 0.50 & -93.5 & 0.5 & 0.49 & 105 \\
\hline 33868 & 561.2357 & 0.296 & -86.3 & 0.8 & 0.15 & +139.3 & 1.4 & -0.80 & 90 \\
\hline 33870 & 561.3171 & 0.339 & -74.5 & 0.8 & -0.68 & +125.2 & 1.0 & -1.30 & 137 \\
\hline 33932 & 564.2289 & 0.871 & +105.2 & 0.6 & -0.20 & -66.2 & 0.8 & -0.37 & 122 \\
\hline 33965 & 564.5032 & 0.015 & +12.8 & 0.5 & 0.72 & +35.3 & 1.7 & 0.98 & 111 \\
\hline 34066 & 570.2604 & 0.044 & -6.5 & 0.5 & 0.80 & +57.8 & 0.3 & 0.60 & 140 \\
\hline 36011 & 894.3444 & 0.530 & +44.4 & 0.6 & 0.23 & -0.8 & 0.3 & -0.68 & 95 \\
\hline 36072 & 895.4091 & 0.090 & -39.8 & 0.9 & -1.53 & +88.1 & 0.8 & -0.25 & 113 \\
\hline 36080 & 895.5091 & 0.143 & -66.9 & 0.7 & -0.52 & +119.0 & 0.7 & 0.50 & 137 \\
\hline 36123 & 896.4234 & 0.624 & +102.7 & 0.7 & -0.20 & -64.2 & 0.8 & -1.06 & 142 \\
\hline 36125 & 896.4457 & 0.636 & +107.8 & 0.7 & -1.00 & -70.8 & 0.9 & -1.35 & 139 \\
\hline 36126 & 896.4658 & 0.646 & +112.9 & 0.5 & -0.40 & -75.6 & 0.9 & -1.29 & 143 \\
\hline 36128 & 896.4883 & 0.658 & +116.7 & 1.0 & -1.60 & -80.9 & 1.0 & -1.26 & 145 \\
\hline
\end{tabular}

described in Paper I exactly, to which we refer for details of the observing mode. Here we recall that the wavelength region covered is 4500-9480 $\AA$ with a resolving power $R \sim 20000$. A journal of the observations is given in Table 2 , in which the first three columns list the spectrum number (from the Asiago Echelle log book), the heliocentric JD (-2451000), and the orbital phase, while the last column gives the $S / N$ per pixel of the recorded stellar continuum averaged over the whole measured wavelength range (4890-5690 ^). The other columns list, for both components separately and in $\mathrm{km} \mathrm{s}^{-1}$, the radial velocity, its error, and the difference with the computed orbit. We obtained 31 spectra with exposure times ranging from 1200 to $1800 \mathrm{~s}$, which guarantee a good $S / N$ ratio (cf. Table 2) while avoiding smearing due to the orbital motion (1500 s correspond to less than $1 \%$ in orbital period).

As outlined in Paper II, small residual spectrograph flexures are removed by cross-correlating the rich telluric absorptions complexes at 5880-5940, 6275-6310, 6865-7050, and 7160-7330 ^ with a synthetic telluric absorption spectrum. Night-sky lines ([OI] and $\mathrm{OH})$ and city lines ( $\mathrm{HgI}$ and $\mathrm{NaI}$ ) are used to check the accuracy of the wavelength calibration. Their mean velocity was found to be $0.0 \pm 0.1 \mathrm{~km} \mathrm{~s}^{-1}$ on every spectrum.

\subsection{Radial velocities}

In deriving the radial velocities of V570 Per, we strictly followed the method outlined in details in Paper I. In short, six adjacent Echelle orders, covering the 4890-5690 $\AA$ wavelength range with no inter-order gaps, are measured via two-dimensional cross-correlation technique (TODCOR), based on the Zucker \& Mazeh (1994) algorithm. These six Echelle orders are chosen because they are densely populated by absorption

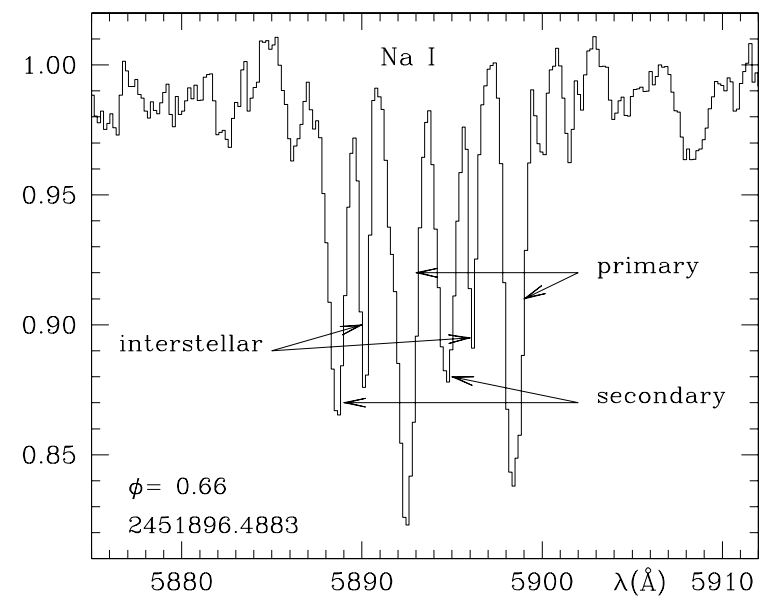

Fig. 2. The NaI doublet $(5890,5896 \AA)$ region for V570 Per. The interstellar components are well-separated from the stellar ones.

lines, in particular FeI and $\mathrm{MgI}$, which perform particularly well in terms of radial velocity. As templates we use synthetic spectra with the appropriate temperatures, surface gravities and rotational velocities, selected from the Munari et al. (2005, hereafter M05) synthetic spectral atlas computed at the same 20000 resolving power as the Echelle scientific spectra. The high reliability of our TODCOR-based radial velocities has been subjected to extensive tests described in Paper II. They performed equally well when repeated here on V570 Per data, and we can conclude that no external systematic error in excess of $0.25 \mathrm{~km} \mathrm{~s}^{-1}$ affects our radial velocities.

The results of the radial velocity measurements are reported in Table 2. The mean error of radial velocities is $0.6 \mathrm{~km} \mathrm{~s}^{-1}$ for star 1 and $0.7 \mathrm{~km} \mathrm{~s}^{-1}$ for star 2, as estimated from comparison of the radial velocities obtained separately from each of the six Echelle orders analyzed here.

\subsection{Reddening}

The measurement of reddening is a key step in determining the absolute temperature scale (and therefore the distance) of eclipsing binaries. In spite of its short distance, some reddening is expected to affect V570 Per given its low galactic latitude $(l=145.18, b=-8.19)$.

Our spectra cover the interstellar NaI (5890 and $5896 \AA$ ) and KI (7665 and $7699 \AA$ ) doublets, which are excellent estimators of the reddening as demonstrated by Munari \& Zwitter (1997). They calibrated a tight relation linking the NaI D2 (5890 ̊) and KI (7699 ̊) equivalent widths with the $E_{B-V}$ reddening. On spectra obtained at quadratures, lines from both components are un-blended with the interstellar ones, which can therefore be accurately measured. An example of such a spectrum is presented in Fig. 2. We derived an equivalent width of $0.08 \pm 0.03 \AA$ for NaI D2 (5890 $\AA$ ), which corresponds to $E_{B-V}=0.023 \pm 0.007 \mathrm{mag}$. This is the value adopted in the rest of the paper. At such low reddening, no detectable KI interstellar line is expected, as confirmed by our spectra.

As mentioned above, V570 Per is seen in the foreground of the $\alpha$ Per cluster. The cluster extends over a wide area and suffers from a patchy distribution of the reddening, which amounts to $E_{B-V}=0.09$ according to Crawford \& Barnes (1974) and $E_{B-V}=0.11$ following Prosser (1992). It is worth noticing that HD 19665, which lies less than 1 arcmin from V570 Per, 
Table 3. Orbital solution for V570 Per. The set of parameters in Col. 2 refer to the unconstrained solution described in Sect. 4, those in Col. 3 to the luminosity-constrained solution of Sect. 6.

\begin{tabular}{|c|c|c|c|c|}
\hline \multirow[b]{2}{*}{$P \quad(\mathrm{~d})$} & \multicolumn{2}{|c|}{ unconstrained } & \multicolumn{2}{|c|}{ luminosity constrained } \\
\hline & 1.9009382 & \pm 0.0000008 & 1.9009382 & \pm 0.0000008 \\
\hline$T_{0}$ (HJD) & 2451895.2371 & \pm 0.0002 & 2451895.2371 & \pm 0.0002 \\
\hline $\mathrm{a} \quad\left(\mathrm{R}_{\odot}\right)$ & 9.0929 & \pm 0.013 & 9.0929 & \pm 0.012 \\
\hline$V_{\gamma}\left(\mathrm{km} \mathrm{sec}^{-1}\right)$ & +22.81 & \pm 0.03 & +22.81 & \pm 0.03 \\
\hline$q=\frac{\mathrm{m}_{2}}{\mathrm{~m}_{1}}$ & 0.9319 & \pm 0.0008 & 0.9319 & \pm 0.0008 \\
\hline i (deg) & 77.44 & \pm 0.34 & 77.44 & \pm 0.32 \\
\hline e & 0 & & 0 & \\
\hline$T_{1}(\mathrm{~K})$ & 6842 & \pm 25 & 6842 & \pm 25 \\
\hline$T_{1}-T_{2}(\mathrm{~K})$ & 262 & \pm 70 & 280 & \pm 35 \\
\hline$\Omega_{1}$ & 7.00 & \pm 0.43 & 6.93 & \pm 0.13 \\
\hline$\Omega_{2}$ & 7.28 & \pm 0.50 & 7.16 & \pm 0.08 \\
\hline $\mathrm{r}_{1}\left(R_{1} / \mathrm{a}\right)$ & 0.1655 & \pm 0.0193 & 0.1675 & \pm 0.0031 \\
\hline $\mathrm{r}_{2}\left(R_{2} / \mathrm{a}\right)$ & 0.1498 & \pm 0.0123 & 0.1526 & \pm 0.0019 \\
\hline$R_{1} \quad\left(\mathrm{R}_{\odot}\right)$ & 1.50 & \pm 0.11 & 1.523 & \pm 0.030 \\
\hline$R_{2} \quad\left(\mathrm{R}_{\odot}\right)$ & 1.36 & \pm 0.11 & 1.388 & \pm 0.019 \\
\hline$M_{1} \quad\left(\mathrm{M}_{\odot}\right)$ & 1.4487 & \pm 0.0062 & 1.4487 & \pm 0.0058 \\
\hline$M_{2} \quad\left(\mathrm{M}_{\odot}\right)$ & 1.3500 & \pm 0.0059 & 1.3500 & \pm 0.0055 \\
\hline $\log g_{1}(\operatorname{cgs})$ & 4.25 & \pm 0.06 & 4.23 & \pm 0.02 \\
\hline $\log g_{2}(\mathrm{cgs})$ & 4.30 & \pm 0.07 & 4.28 & \pm 0.01 \\
\hline $\mathrm{M}_{\mathrm{bol}, 1}$ & 3.13 & \pm 0.17 & 3.11 & \pm 0.05 \\
\hline $\mathrm{M}_{\mathrm{bol}, 2}$ & 3.54 & \pm 0.18 & 3.49 & \pm 0.04 \\
\hline$d_{\text {orb }} \quad(p c)$ & 121 & \pm 10 & 123 & \pm 2 \\
\hline$d_{\mathrm{HIP} 1997}(\mathrm{pc})$ & 117 & \pm 14 & & \\
\hline$d_{\mathrm{HIP} 2007}(\mathrm{pc})$ & 123 & \pm 11 & & \\
\hline
\end{tabular}

is affected by $E_{B-V}=0.022$ reddening, according to Perry \& Johnston (1982).

\section{Orbital solution}

The orbital modeling is performed with version WD98K93 (Milone et al. 1992) of the Wilson-Devinney code (Wilson \& Devinney 1971; Wilson 1998), which incorporates modified stellar atmospheres. The linear limb darkening coefficients are taken from van Hamme (1993) for the appropriate $T_{\text {eff }}, \log g$, $[\mathrm{M} / \mathrm{H}]$. The temperature of the primary adopted in the orbital solution, $T_{1}=6842 \pm 25 \mathrm{~K}$, was determined by atmospheric analysis (see Sect. 5 below). It is in close agreement with indications from spectral classification and de-reddened colors. Comparing the spectrum of V570 Per in the 8480-8740 A wavelength region with the Gaia spectral atlas of Munari \& Tomasella (1999, as compiled from observations of MK standards obtained with the same instrument and resolution of the V570 Per spectra discussed in this paper), a spectral classification $\mathrm{F} 3 \mathrm{~V}+\mathrm{F} 5 \mathrm{~V}$ is derived. The temperature for the F3V primary is $6845 \mathrm{~K}$, according to Bertone et al. (2004) calibration. The color out of eclipse is $(B-V)=+0.452( \pm 0.005)$, that corrected for $E_{B-V}=0.023$ $( \pm 0.007)$, provides $(B-V)_{0}=+0.429( \pm 0.009)$. In comparison with Drilling \& Landolt (2000) color calibration for main sequence stars, it corresponds to a spectral type F4V, i.e. the mean spectral type of the two components of the binary.

The Gaia-like orbital solution of M01 was adopted as the set of initial values for input parameters in the orbital modeling: period $P$, epoch $T_{0}$, semi-major axis $a$, barycentric radial velocity $V_{\gamma}$, mass ratio $q=M_{1} / M_{2}$, inclination $i$, eccentricity $e$, modified Kopal potentials $\Omega_{1,2}$, and $L_{1} / L_{2}$ ratio in each pass-band.

We computed the solution reported in the second column of Table 3 (unconstrained) using mode 2 of the Wilson-Devinney code, which is appropriate for detached binaries, with no constraints on the potentials and on the luminosity ratio of the two components. The adopted bolometric albedos and exponents in the bolometric gravity brightening law were set to $A_{1}=A_{2}=0.5$ and $g_{1}=g_{2}=0.3$, respectively, as appropriate for convective atmospheres (Wilson 1998). As in Paper II, full orbital solution runs were also carried out with logarithmic and square-root limb darkening laws, as well as with various combinations of $A_{1,2}$ and $g_{1,2}$ in the range $0.5 \leq A_{1,2} \leq 1.0$ and $0.3 \leq g_{1,2} \leq 1.0$ ( $A$ and $g$ are expected to be close to unity for radiative envelopes). No improvement in the formal accuracy of the solution was obtained. No orbital parameter was found to vary, as a result of these extensive experiments, by more than its formal error listed in the orbital solution of Table 3. The latter was obtain with the following parameters for the linear limb darkening law: $x_{\text {bol, } 1}=0.607, x_{\text {bol, } 2}=0.664, x_{V, 1}=0.407, x_{V, 2}=0.428$, $x_{B, 1}=0.504, x_{B, 2}=0.525$. No evidence was found for multiple reflection effects or a third light presence.

The orbital period of V570 Per is stable. Our photometry provides five epochs of minima (all of them with an uncertainty of \pm 0.00012 days): primary eclipses on HJD $=2451895.23713$, $2451920.44464,2452986.37566$, and secondary ones on HJD $=2451951.31480,2452$ 158.51707. The Hipparcos and Tycho Catalogues (ESA 1997) give $2448500.1520 \pm 0.001$ as the time of primary minimum, revised to $2448500.1655 \pm 0.003$ in M01 re-analysis in combination with radial velocities. All these minima are well-fitted by the $1.9009382 \pm 0.0000008$ day period of the orbital solution in Table 3.

The V570 Per light and radial velocity curves do not show any signature of an eccentric orbit. The circularity of the orbit is confirmed by initial modeling runs during which eccentricity was allowed to vary; nevertheless, it did not drift away from zero. After a few such trials, $e$ was set to zero for the rest of orbital modeling.

The unconstrained orbital solution of Table 3 is over-plotted to the observational data in Fig. 1. Their rms is 0.006 mag for both $B$ and $V$ photometry, $0.8 \mathrm{~km} \mathrm{~s}^{-1}$ for radial velocities. The formal accuracy on the mass of the primary and secondary is $0.41 \%$ and $0.44 \%$, and on their radius it is $7.3 \%$ and $8.1 \%$, respectively. The uncertainty on the radii is inflated by the low orbital inclination of the system $\left(i=77.44^{\circ} \pm 0.34\right)$, that produces grazing eclipses of low amplitude $\left(\Delta B_{\mathrm{I}}=0.116, \Delta B_{\mathrm{II}}=0.094\right.$, $\left.\Delta V_{\mathrm{I}}=0.117, \Delta V_{\mathrm{II}}=0.098 \mathrm{mag}\right)$.

As a test, separate orbital solutions were carried out combining in turn $B$ or $V$ photometry alone with the radial velocities. We converged to the same orbital parameters with larger formal errors, as expected. Furthermore, the orbital solution was carried out on $B$ and $V$ photometric data together, without including radial velocities in the fitting process, and retaining $i, M_{1}, M_{2}$ from the final solution of Table 3 (unconstrained). Again, the orbital solution converged - within the formal errors - to the same set of parameters as are given in Table 3, with $R_{1}=1.55 \pm 0.02 R_{\odot}$ (instead of $1.50 \pm 0.11$ ) and $R_{2}=1.33 \pm 0.02 R_{\odot}$ (instead of $1.36 \pm 0.11)$.

To check the accuracy of the derived orbital solution, we compared the corresponding distance with the Hipparcos parallax. In calculating the distance, we adopted a bolometric magnitude $M_{\mathrm{bol}, \odot}=4.74$ from Bessell et al. (1998) for the Sun and, for the corresponding atmospheric parameters, a bolometric correction $B C=0.00$ for both V570 Per components. The resulting distance, for the $E_{B-V}=0.023$ color excess derived in Sect. 3.2 , is $d_{\text {orb }}=121 \pm 10$ pc, in agreement with the $123 \pm$ 11 pc revised Hipparcos parallax for V570 Per (van Leeuwen 2007 , and private communication in advance of publication). 

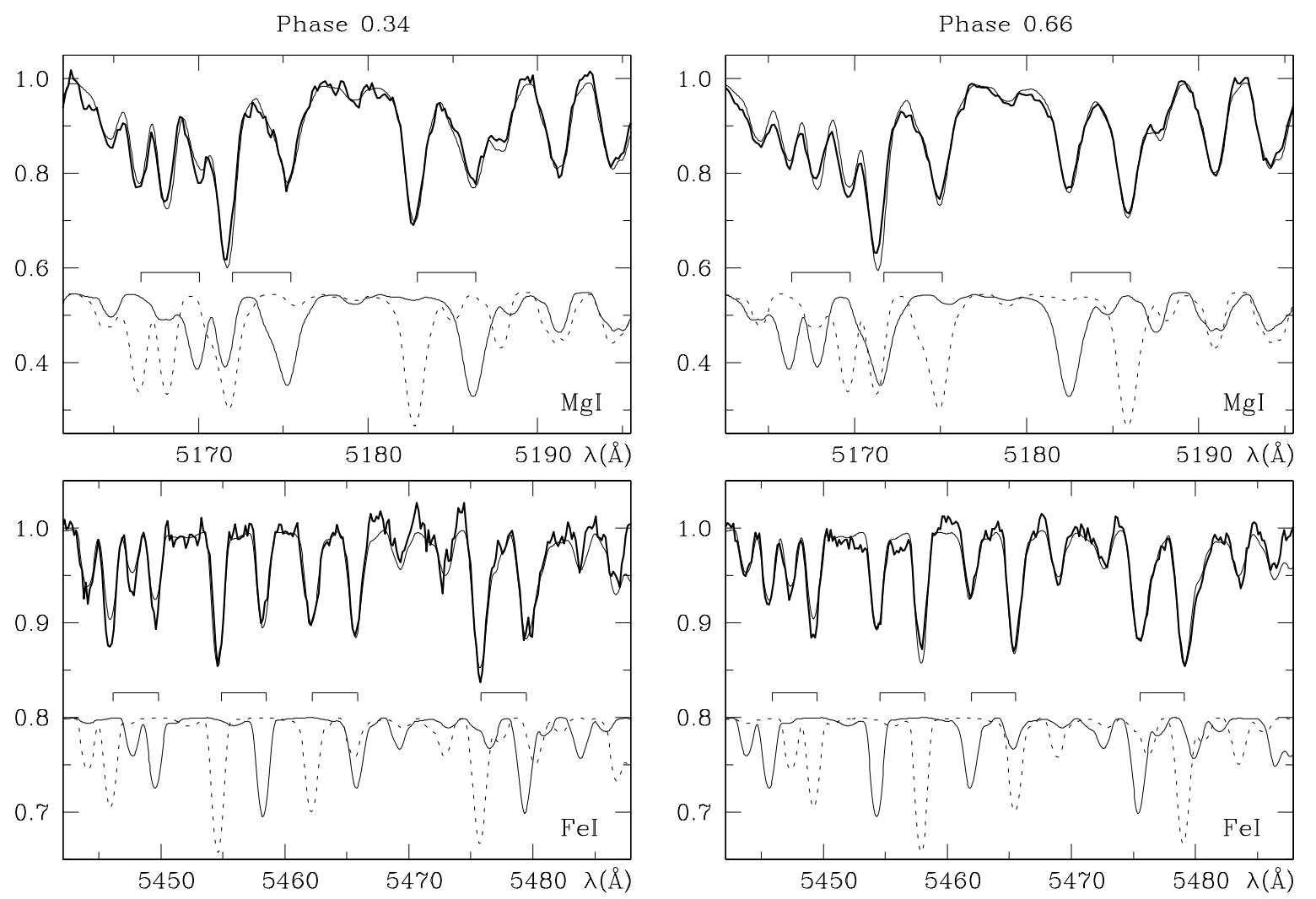

Fig. 3. Comparison between observed (thick line) and synthetic (thin line) V570 Per spectra over two sample wavelength regions dominated by MgI lines (top) and FeI lines (bottom). Spectra at orbital phases 0.34 and 0.66 (\# 33870 and 36128 in Table 2, respectively) are shown, which allows full and reversed splitting of the absorption lines of the two components. In each panel the lower curves represent (not to scale with the main spectra but in correct proportion between them) the contribution of each component of the binary to the formation of the observed spectrum at the given phase. The markers connect the shifted wavelengths of the same MgI (top panels) and FeI (bottom panels) lines in the spectra of the two components of the binary.

Varying the adopted value for the solar bolometric magnitude (e.g. from $M_{\text {bol, } \odot}=4.72$ reported by Straizys \& Kuriliene 1981 , to $M_{\text {bol, } \odot}=4.74$ by Livingston 2000 , and $M_{\text {bol, } \odot}=4.83$ by Popper 1980) and the corresponding bolometric correction for F5V stars $(B C=-0.02,-0.08$ and -0.03 , respectively), the distance derived from the orbital solution changes by $\pm 2 \mathrm{pc}$.

\section{Atmospheric analysis}

To derive the atmospheric parameters of both V570 Per components, we performed a $\chi^{2}$ fit against the synthetic spectral atlas of M05. It covers the 2500-10500 $\AA$ wavelength range at the same resolving power as our spectra $(R=20000)$. The M05 synthetic spectra were calculated with the revised solar abundances by Grevesse \& Sauval (1998) and the new opacity distribution functions (ODFs) of Castelli \& Kurucz (2004) throughout the whole HR diagram for 12 different rotational velocities, $T_{\text {eff }}$ ranging from 3500 to $47500 \mathrm{~K}, \log g$ from 0.0 to 5.0 , [M/H] from -2.5 to +0.5 , two values of $\alpha$-enhancement $([\alpha / \mathrm{Fe}]=0.0$ and +0.4$)$, and three micro-turbulent velocities $\left(1,2,4 \mathrm{~km} \mathrm{~s}^{-1}\right)$. The $\chi^{2}$ fitting was restricted to the higher $S / N$ spectra obtained at both quadratures. We limited the fitting to the same 4890-5590 wavelength range as was used to derive the radial velocities.

In the $\chi^{2}$ fitting procedure, we fixed only the radial velocities of the two components, while $T_{\text {eff }}, \log g, V_{\text {rot }} \sin i,[\mathrm{M} / \mathrm{H}]$, micro-turbulent velocity, and the brightness ratio for both components were treated as free parameters. The results are given in Table 4. The listed errors are the error of the mean computed on
Table 4. Atmospheric parameters (and their error of the mean, cf. Sect. 5) of V570 Per from the $\chi^{2}$ fit to synthetic spectra in comparison to the results from orbital solution.

\begin{tabular}{|c|c|c|c|c|}
\hline \multirow[b]{2}{*}{$T_{\text {eff }, 1}(\mathrm{~K})$} & \multicolumn{2}{|c|}{$\begin{array}{c}\chi^{2} \text { fit to } \\
\text { synth. spectra }\end{array}$} & \multicolumn{2}{|c|}{ orbital solution } \\
\hline & 6842 & \pm 25 & & \\
\hline$T_{\mathrm{eff}, 2}(\mathrm{~K})$ & 6562 & \pm 25 & & \\
\hline$\Delta T_{\mathrm{eff}}=T_{\mathrm{eff}, 1}-T_{\mathrm{eff}, 2}(\mathrm{~K})$ & 280 & \pm 35 & 262 & \pm 70 \\
\hline $\log g_{1}(\mathrm{cgs})$ & 4.25 & \pm 0.13 & 4.25 & \pm 0.05 \\
\hline $\log g_{2}(\mathrm{cgs})$ & 4.25 & \pm 0.13 & 4.30 & \pm 0.05 \\
\hline$V_{\mathrm{rot}, 1} \sin i\left(\mathrm{~km} \mathrm{sec}^{-1}\right)$ & 40 & \pm 5 & 40 & \pm 1 \\
\hline $\begin{array}{l}V_{\text {rot,2 }} \sin i\left(\mathrm{~km} \mathrm{sec}^{-1}\right) \\
{[\mathrm{M} / \mathrm{H}]}\end{array}$ & $\begin{array}{r}40 \\
+0.02\end{array}$ & $\begin{array}{l} \pm 5 \\
\pm 0.03\end{array}$ & 36 & \pm 1 \\
\hline $\mathrm{L}_{V, 1} /\left(\mathrm{L}_{V, 1}+\mathrm{L}_{V, 2}\right)$ & 0.60 & & 0.59 & \\
\hline $\mathrm{L}_{V, 2} /\left(\mathrm{L}_{V, 1}+\mathrm{L}_{V, 2}\right)$ & 0.40 & \} \pm 0.02 & 0.41 & \} \pm 0.12 \\
\hline
\end{tabular}

the results obtained separately on the six Echelle orders covering the 4890-5590 $\AA$ wavelength range. Table 4 also provides a comparison with parameters in common with the orbital solution, and it fosters strong confidence in the results of the atmospheric analysis. Figure 3 highlights the goodness of the match between observed and synthetic spectra by over-plotting sample wavelength intervals of them.

In a configuration such as that of V570 Per, with similar stars and partial eclipses that are relatively shallow, the information on 
the relative sizes of the stars is weak. This reflects on the appreciable uncertainties of the radii in Table 3 (second column, unconstrained solution). The brightness ratio of the two stars as determined by the $\chi^{2}$ fit offers an important and independent check on the accuracy of the radii. Such a comparison is provided in Table 4 as brightness ratio of the two stars. It is carried out in the $V$ band because the six contiguous Echelle orders used in our atmospheric analysis are centered at its effective wavelength. The brightness ratios derived by $\chi^{2}$ fit and orbital solution are the same, well within the respective errors. Also the TODCOR algorithm converged on the same $0.60 / 0.40$ brightness ratio when deriving the radial velocities of the two components.

The V570 Per components rotate in synchronicity with the orbital motion (as highlighted by the comparison between observed and expected $V_{\text {rot }} \sin i$ in Table 4), have spherical shapes $\left(R_{\mathrm{L} 1}=R_{\text {pole }}\right)$, and are widely separated $(R / a \sim 0.16)$. Under these circumstances, no tidally induced chromospheric activity is expected. This is confirmed by the absence of emission line cores or emission veiling in the CaII H \& K blue doublet or CaII far-red triplet lines, as verified at all orbital phases by comparison with synthetic spectra.

\section{Constraining the stellar radii}

The shallow eclipses displayed by V570 Per cannot sharply constrain the stellar radii, as indicated by the $\sim 8 \%$ error on the radii obtained in the unforced orbital solution of Sect. 4 , which is listed in the second column of Table 3. However, a relevant constraint is provided by the luminosity ratio of the two components as determined by $\chi^{2}$ analysis and TODCOR fitting (cf. Sect. 5 and last two lines of Table 4). We therefore re-ran the orbital solution with the Wilson-Devinney code, operated this time in mode 0 , which is specific to the case when the luminosities are externally supplied. We then converged to the luminosityconstrained orbital solution listed in the third column of Table 3. It confirms the unforced orbital solution of Sect. 4, with an overall decrease in formal errors and in particular in those on stellar radii and on the distance to the binary system. The radii improved from $1.50 \pm 0.11,1.36 \pm 0.11 R_{\odot}$ to $1.523 \pm 0.030$, $1.388 \pm 0.019 R_{\odot}$, and the distance to the system from $121 \pm 10$ to $123 \pm 2 \mathrm{pc}$. The last is now identical to the revised Hipparcos distance $(123 \pm 11 \mathrm{pc}$, van Leeuwen 2007).

The comparison of V570 Per with theoretical stellar models carried out in Sect. 7 and in Figs. 4-6 is based on this luminosity-constrained orbital solution.

\section{Observations vs. theory}

As extensively reviewed in Paper II, binary systems with wellmeasured masses, radii, effective temperatures, and heavyelement abundance provide stringent tests of evolutionary stellar models. Many issues can be investigated by comparing empirical evidence for the binary stars and theoretical predictions such as the efficiency of super-adiabatic convection in low-mass stars, the efficiency of diffusive processes (atomic diffusion and radiative levitation), and the real extension of convective core overshooting during the central H-burning stage. For intermediate mass stars, i.e. stars more massive than about $1.2 M_{\odot}$, this issue is really a longstanding problem (see e.g. Cassisi 2004, and references therein). An additional, still unsettled issue is how convective core overshooting reduces in response to a decrease in stellar mass and its canonical convective core (see Woo \& Demarque 2001, for a detailed discussion).

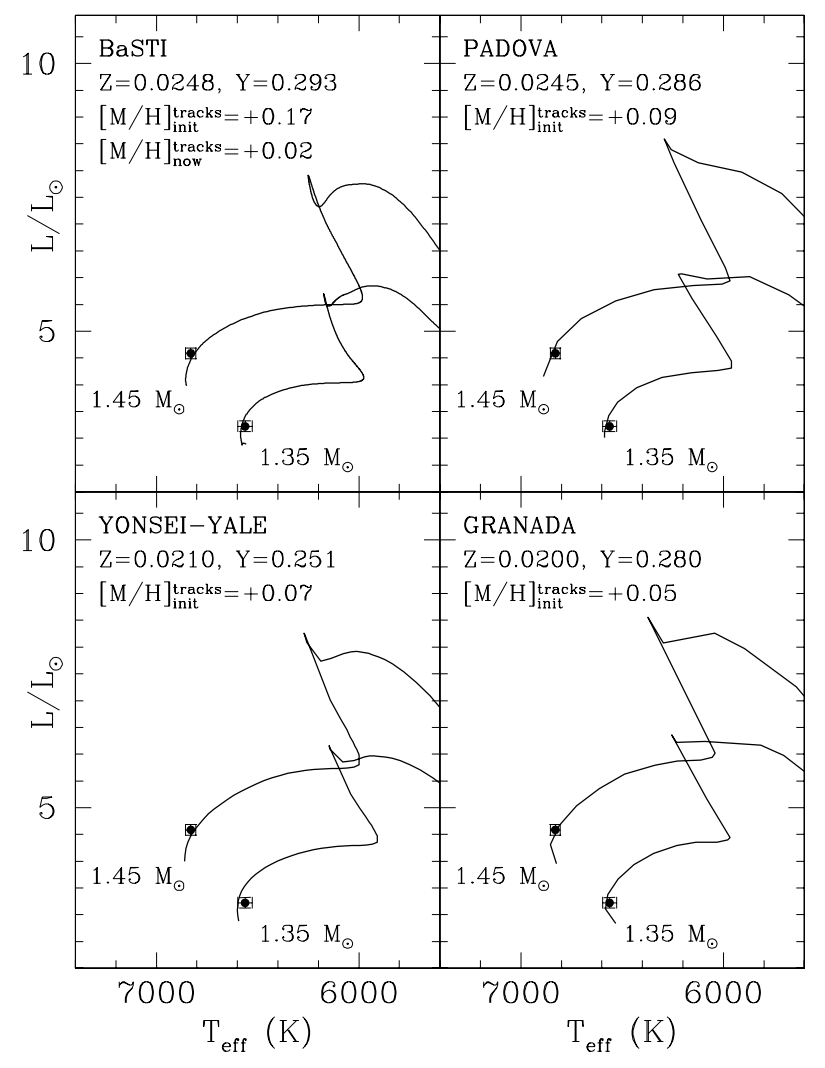

Fig. 4. Comparison of evolutionary tracks with observed parameters for V570 Per components (filled circles with error bars). The metallicity, helium content, and mass of the fitting tracks are given in each panel. Padova, Yonsei-Yale, and Granada tracks are interpolated from published ones, while BaSTI tracks have been computed on purpose for this work for the exact masses of the two components (1.4487 and $\left.1.3500 M_{\odot}\right)$.

In Paper II, we have briefly outlined the main results that were obtained in this context by using empirical data for galactic open clusters of suitable age. We noticed the strong limitations imposed by the uncertainties in the distance, reddening, and heavy-element abundances adopted for the clusters (cf. Vandenberg et al. 2007). A firmer contribution can be provided by eclipsing binary systems of suitable masses, with examples provided by V459 Cas (Sandberg Lacy et al. 2004), TZ For (Vandenberg et al. 2006), AI Phe (Andersen et al. 1988; Pietrinferni et al. 2004, hereafter P04), and V505 Per (Paper II). The above derived $1.449 \pm 0.006 M_{\odot}$ and $1.350 \pm 0.006 M_{\odot}$ masses for the two components of V570 Per qualify this binary too as a suitable constraint on stellar theoretical models so worth a detailed discussion.

The V570 Per measured properties were compared with the following sets of theoretical stellar models: BaSTI (P04, see also Cordier et al. 2007), Padova (Girardi et al. 2000), YonseiYale (Yi et al. 2001, second release), and Granada (Claret et al. 2003), which have all been computed by assuming a scaledsolar, heavy-element distribution.

The loci of theoretical isochrones and of V570 Per components on the HR diagram are shown in Fig. 4. For Yonsei-Yale, Granada, and Padova models, we interpolated linearly both in mass and metallicity between the closest published isochrones. BaSTI models, on the other hand, have been computed on purpose for this paper, for exactly the measured masses of the two V570 Per components and exploring a fine grid in metal abundance, helium content, element diffusion, and amount of 


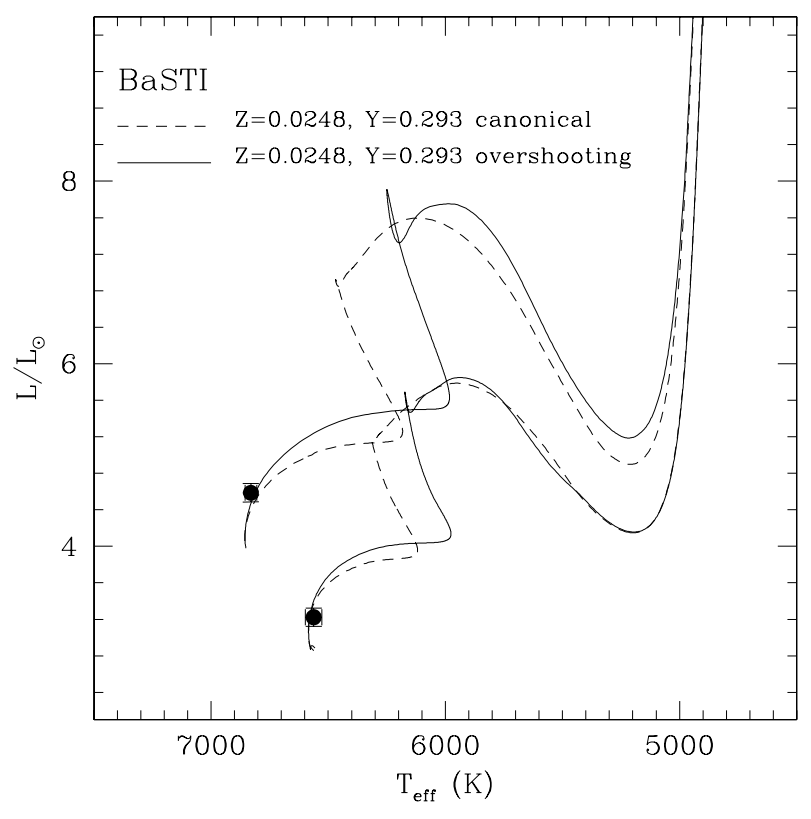

Fig. 5. Comparison of V570 Per components with BaSTI tracks computed - for the exact observed masses - with and without overshooting (see Sect. 6 for details).

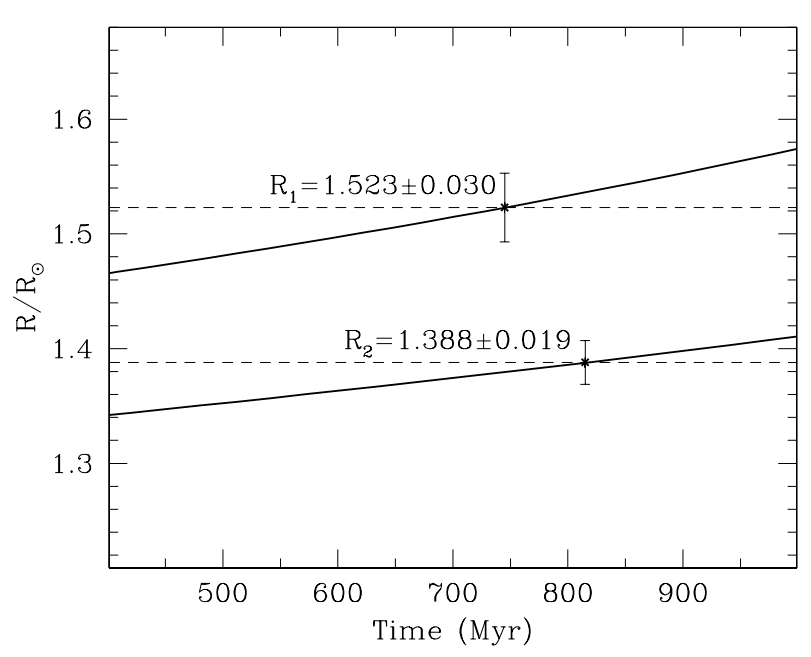

Fig. 6. Comparison between the observed radii for the V570 Per components (dashed lines), their uncertainty (error bars), and the BaSTI evolutionary tracks (solid lines) computed with overshooting and for exactly the observed individual masses. The resulting age for V570 Per is $\approx 790 \pm 60 \mathrm{Myr}$.

core overshooting (including canonical models with no overshooting). The overshooting was treated in the same way as in Paper II, as extensively discussed by P04.

The Padova and Granada models do not account for element diffusion during the life of a star, and Yonsei-Yale does it only for helium. Thus, the metallicity of their fitting isochrones in Fig. 4 pertains to the initial composition when V570 Per was born and not to its present value at the stellar surface. It is therefore expected that the metallicities derived by fitting to Padova, Granada, and Yonsei-Yale isochrones are higher than measured by spectroscopy. The best-fitting BaSTI models have an initial metallicity of $[\mathrm{M} / \mathrm{H}]=+0.17$ for both stars, which decreases to $[\mathrm{M} / \mathrm{H}]=+0.00$ and +0.04 for the 1.45 and $1.35 M_{\odot}$ components, respectively, by the time of V570 Per current 0.8 Gyr age (see below for its derivation). The average of this values compares very well with the spectroscopic measurement of $[\mathrm{M} / \mathrm{H}]=$ $+0.02 \pm 0.03$ for the V570 Per components.

The comparison with theoretical isochrones in Fig. 4 shows that both components of V570 Per are only marginally evolved. The more massive of the two stars has already moved slightly away from the zero age main sequence. Figure 5 shows the effect of overshooting, which has been computed for BaSTI models according to the same $\mathrm{P} 04$ prescriptions already adopted in Paper II. This figure shows that canonical stellar models with no overshooting, but accounting for element diffusion, can still reproduce the position of V570 Per in the HR diagram (dashed line in Fig. 5). However, the canonical models give a surface metallicity at the present age of the binary system equal to $[\mathrm{M} / \mathrm{H}]=+0.12$, which is outside the range of uncertainty for the observed $[\mathrm{M} / \mathrm{H}]=+0.02 \pm 0.03$ value. On the other hand, a small amount of overshooting allows a perfect fit for both the spectroscopic metallicity and the HR diagram location, as illustrated by the solid lines in Fig. 5, which have been computed for an efficiency of the overshooting amounting to $\lambda_{\mathrm{OV}}=0.14$ for the $1.449 \pm 0.006 M_{\odot}$ primary and $\lambda_{\mathrm{OV}}=0.11$ for the $1.350 \pm 0.006 M_{\odot}$ secondary. In Paper II, for the lighter components of V505 Per, we obtained proportionally lower efficiencies: $\lambda_{\mathrm{OV}}=0.093$ and 0.087 for the 1.269 and $1.251 M_{\odot}$ components, respectively. The efficiency is usually defined in terms of the parameter $\lambda_{\mathrm{OV}}$ that gives the length - expressed as a fraction of the local pressure scale height $H_{\mathrm{P}}$ - crossed by the convective cells in the convective stable region outside the Schwarzschild convective boundary. Regardless of the initial metallicity, P04 adopt $\lambda_{\mathrm{OV}}=0.20 \times H_{\mathrm{P}}$ for $M \geq 1.7 M_{\odot}, \lambda_{\mathrm{OV}}=0$ for $M \leq 1.1 M_{\odot}$, and $\lambda_{\mathrm{OV}}=\left(\frac{M}{M_{\odot}}-0.9\right) / 4$ for $1.1 M_{\odot} \leq M \leq 1.7 M_{\odot}$.

To estimate the age of the system, in Fig. 6 we plot the evolution in radius of the BaSTI models above described for the two V570 Per components and compare them with the observed values. The derived system age is $790 \pm 60 \mathrm{Myr}$.

Acknowledgements. We would like to thank the anonymous referee for valuable suggestions, R. Barbon and E. Nasi for useful discussion, F. van Leeuwen who kindly communicated the revised Hipparcos parallax of V570 Per in advance of publication, and the technical staff operating the $1.82 \mathrm{~m}$ telescope in Asiago for the skillful assistance during the whole project.

\section{References}

Andersen, J., Clausen, J. V., Gustafsson, B., Nordström, B., \& Vandenberg, D. A. 1988, A\&A, 196, 128

Bertone, E., Buzzoni, A., Chavez, M., \& Rodriguez-Merino, L. H. 2004, AJ, 128,829

Bessell, M. S. 2000, PASP, 112, 961

Bessell, M. S., Castelli, F., \& Plez, B. 1998, A\&A, 333, 231

Cassisi, S. 2004, in Variable Stars in the Local Group, IAU Colloq. 193, Christchurch (New Zealand), ed. D. W. Kurtz, \& K. R. Pollard, ASP Conf. Proc., 310, 489

Castelli, F., \& Kurucz, R. L. 2004, A\&A, 419, 725

Claret, A., Paunzen, E., \& Maitzen, H. M. 2003, A\&A, 412, 91

Cordier, D., Pietrinferni, A., Cassisi, S., \& Salaris, M. 2007, AJ, 133, 468

Crawford, D. L., \& Barnes J. V. 1974, AJ, 79, 687

Drilling, J. S., \& Landolt, A. U. 2000 in Allen's Astrophysical Quantities, ed. A. N. Cox (Springer), 381

ESA 1997, The Hipparcos and Tycho Catalogues, ESA SP-1200

Girardi, L., Bressan, A., Bertelli, G., \& Chiosi, C. 2000, A\&AS, 141, 371

Grevesse, N., \& Sauval, A. J. 1998, Space Sci. Rev., 85, 161

Lang, K. R. 2006, in Astrophysical Formulae, 3rd edn. (Springer)

Livingston, W. C. 2000, in Allen's Astrophysical Quantities, ed. A. N. Cox (Springer), 339

Milone, E. F., Stagg, C. R., \& Kurucz, R. L. 1992, ApJS, 79, 123 
Munari, U., \& Zwitter, T. 1997, A\&A, 318, 269

Munari, U., \& Tomasella, L. 1999, A\&AS, 137, 521

Munari, U., Tomov, T., Zwitter, T., et al. 2001, A\&A, 378, 477

Munari, U., Sordo, R., Castelli, F., \& Zwitter, T. 2005, A\&A, 442, 1127

Perry, C. L., \& Johnston, L. 1982, ApJ, 50, 451

Popper, D. M. 1980, ARA\&A 18, 115

Pietrinferni, A., Cassisi, S., Salaris, M., \& Castelli, F. 2004, ApJ, 612, 168 Prosser, C. F. 1992, AJ, 103, 488

Sandberg Lacy, C. H., Claret, A., \& Sabby, J. A. 2004, AJ, 128, 132

Siviero, A., Munari, U., Sordo, R., et al. 2004, A\&A, 417, 1083 (Paper I)

Straižys, V., \& Kuriliene, G. 1981, Ap\&SS, 80, 353

Tomasella, L., Munari, U., Siviero, A., et al. 2008, A\&A, 480, 465 (Paper II)
Vandenberg, D. A., Bergbush, P. A., \& Dowler, P. D. 2006, ApJS, 162, 375

Vandenberg, D. A., Gustafsson, B., Edvardsson, B., Eriksson, K., \& Ferguson, J. 2007, ApJL, in press

van Leeuwen, F. 1999, A\&A, 341, L71

van Leeuwen, F. 2007, Hipparcos, the new reduction (Springer)

van Hamme, W. 1993, AJ, 106, 2096

Wilson, R. E. 1998, Computing Binary Star Observables, Univ. of Florida Astronomy Dept.

Wilson, R. E., \& Devinney, E. J. 1971, ApJ, 166, 605

Woo, J.-H., Demarque, P. 2001, AJ, 122, 1602

Yi, S., Demarque, P., Lejeune, T., \& Barnes, S. 2001, ApJS, 136, 417

Zucker, S., \& Mazeh, T. 1994, ApJ, 420, 806 